\section{Nondestructive Dry-matter Estimation of Rose Shoot Leaves, Stems, and Flower Buds Using Regression Models}

\author{
Claudio C. Pasian' and J. Heinrich Lieth ${ }^{2}$ \\ Department of Environmental Horticulture, University of California, Davis, \\ CA 95616-8587
}

Additional index words. Rosa hybrida 'Cara Mia'

\begin{abstract}
Nondestructive dry-weight (DW) estimates of plant parts are important for analyzing production and partitioning patterns of horticultural crops, particularly when repeated measurements of the same plant must be made without affecting growth. Equations were developed for estimating leaf, stem, and flower bud DW (LDW, SDW, and FDW, respectively) from linear measurements of the flowering shoot parts of Rosa hybrida L. 'Cara Mia'. We used a stepwise forward polynomial regression to develop a set of equations that represented the data well; from these, we chose equations to make data collection as simple as possible. LDW was computed from leaf length. LDW of the whole shoot was calculated by adding the computed LDW of each leaf on a shoot. Each stem was divided into 30-mm segments and the DW of each segment was correlated with its diameter. SDW was calculated by adding all of the stem segments' DWs. FDW was directly correlated with flower bud diameter. The selected models can be used for rose shoot DW prediction; although in some cases, errors were encountered. Despite these errors, this approach may represent the only feasible method for DW estimation when destructive methods cannot be used.
\end{abstract}

The manipulation of dry matter distribution among parts of crop plants is an important horticultural element. For example, many crops are pruned regularly to regulate dry matter distribution because this allows some control over carbohydrate partitioning. Understanding dry-matter partitioning is fundamental to optimizing any production system. Studying partitioning patterns involves harvesting and drying plant material, which is time consuming and destructive to the plant. This is especially a problem when performing growth analyses where plants must be sampled frequently.

Most methods of dry-matter distribution analysis are either destructive or expensive. Drying and weighing tissue is obviously too destructive to track individual plants overtime. Methods involving radioactive labeling with ${ }^{14} \mathrm{C}$ can be used to study carbon partitioning, but because" such methods require that small amounts of plant tissue be removed, they are also partially destructive. Although nondestructive, ${ }^{1 "} \mathrm{C}$ labeling is expensive and can be used only over a short time and in the vicinity of a ${ }^{11} \mathrm{C}$ source.

An alternative to these methods is the use of regression models (Ondark, 1971) to correlate plant-part dry weights (DWS) with vari-

Received for publication 9 Apr. 1993. Accepted for publication 20 Oct. 1993, This project was funded in part by a grant from the Joseph H. Hill Foundation and Roses Inc. The cost of publishing this paper was defrayed in part by the payment of page charges. Under postal regulations, this paper therefore must be hereby marked advertisement solely to indicate this fact.

'Postdoctoral Research Associate.

${ }^{2}$ Associate Professor and Extension Specialist. ous dimensions of intact plant parts (e.g., length, width, and diameter). Such models may be applied using measurements obtained without damaging test plants. The drawback to this method is that plant-to-plant variability can cause prediction errors. In addition, partitioning is affected by environmental factors such as light and fertility (Peace and Grubb, 1982). Consequently, regression models generally are valid only for environments similar to those used for parameter estimation. Despite their deficiencies, regression models may be the only reasonable approach for dry-matter estimation when, as in commercial settings, destructive methods cannot be used.

Our objective was to develop a nondestructive, inexpensive method for determining dry matter distribution in cut-flower rose shoots at several development stages. Our focus was on models cot-relating plant-part DWS with various, easily measured dimensions and on characterizing the reliability of such models.

\section{Materials and Methods}

A 2-year-old crop of Rosa hybria 'Cara Mia' was cultivated in a redwood barkamended soil in greenhouse ground beds at the Dept. of Environmental Horticulture, Univ. of California, Davis. Plants were irrigated three times a week with a half-strength Hoagland's solution number 2 plus micronutrients (Hoagland and Amen, 1950). A total of 90 flowering shoots were measured during July and Aug. 1989. Shoots, from the upper part of the rose, were selected at random and at various developmental stages. They ranged from 50 to $750 \mathrm{~mm}$ in length and showed the potential of a commercially acceptable cut flower. The youngest shoots harvested had one un- folded leaf; shoots ready for harvest had a maximum of 17 unfolded leaves. Whenever a shoot was harvested, a section of the stem, including the shoot's two lowest leaves, was left on the mother shoot. A total of 1004 leaves, ranging from small to full size, was measured.

Leaf length (L) was the distance from the petiole-stem juncture to the tip of the terminal leaflet. Leaf width (W) was the maximum width perpendicular to the rachis. After these measurements were taken, each leaf was detached, placed in a small paper bag, and dried at $75 \mathrm{C}$ for 2 days in a drying oven before the leaf DW (LDW) was recorded. A shoot's total LDW (TLDW) was calculatedly adding all of a shoot's LDW values.

Perhaps the simplest model for estimating stem DW (SDW) is one that correlates stem length with DW. This option was discarded because the upper segment of developing shoots is covered by developing leaves, making length measurement impossible. The error of length estimation for young developing shoots can be substantial. We explored an alternative SDW prediction method using stem diameter (S) measurements. We chose eight flowering shoots of various sizes, and mea-

Table 1. Regression models for leaf dry weight $\left(\mathrm{LDW}_{\mathrm{i}}\right)$, stem section dry weight $\left(\mathrm{SDW}_{\mathrm{i}}\right)$, flower dry weight (FDW), and their coefficients of determination.

\begin{tabular}{lc}
\hline \hline Equation & $r^{2}$ \\
\hline LDW $_{\mathrm{i}}=0.0338-0.00195 \mathrm{~L}_{\mathrm{i}}+$ & \\
$0.000039 \mathrm{~L}_{\mathrm{i}}^{2}$ & 0.91 \\
$\mathrm{SDW}$ & $=0.0091+0.00519 \mathrm{~S}_{\mathrm{i}}^{2}$ \\
$\mathrm{FDW}=0.0449-0.00481 \mathrm{~F}^{2}+$ & 0.88 \\
$0.000736 \mathrm{~F}^{3}-16.7 \times 10^{-6} \mathrm{~F}^{4}$ & 0.95 \\
\hline
\end{tabular}
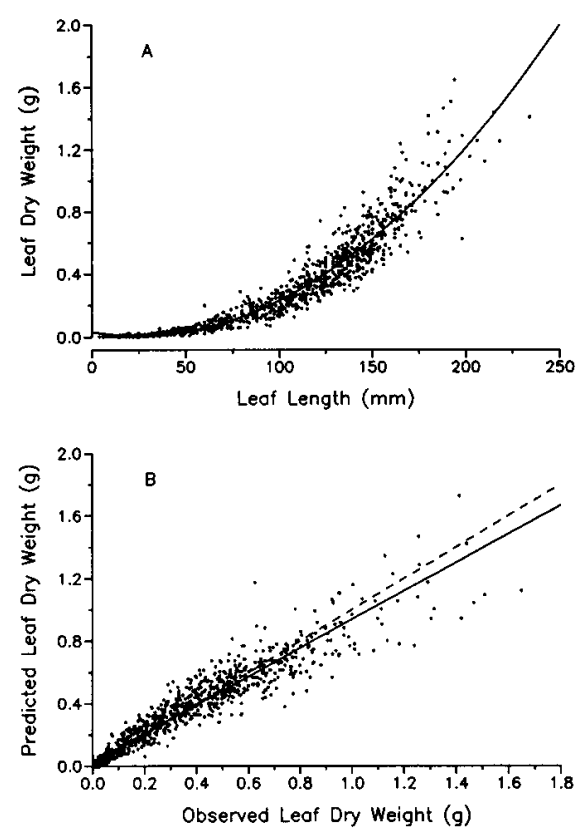

Fig, 1. (A) Observed (dots) and predicted (solid line) leaf dry weights $\left(\mathrm{LDW}_{\mathrm{i}}\right)$ as a function of leaf length and (B) predicted vs. observed LDWs, The dashed line represents the 1:1 correspondence and the solid line represents the linear regression of the predictions: Pred $=0.029+$ 0.909 Obs, 
sured $\mathrm{S}$ at $30-\mathrm{mm}$ intervals along the stem with electronic calipers (Craftsman, Sears Roebuck, Chicago). We cut the defoliated stems into segments at the points where the diameters were measured. The SDW of the segment $\left(\mathrm{SDW}_{\mathrm{i}}\right)$ and $\mathrm{S}$ of the segment $\left(\mathrm{S}_{\mathrm{i}}\right)$ were recorded (202 observations). SDW was calculated by adding a shoot's $\mathrm{SDW}_{\mathrm{i}}$.

A total of 62 flower buds at various development stages were selected from the flower bud population. Diameters of the bud (F) were measured with the same electronic calipers used to measure $\mathrm{S}$. There were few buds with diameters $<2.9 \mathrm{~mm}$ because such buds were protected by developing leaves. Before determining the flower DW (FDW), each flowering bud was oven-dried.

Equations characterizing the three data sets were developed with stepwise forward regression using the SAS REG/Stepwise procedure (SAS Institute, 1987). Model suitability was evaluated using goodness-of-fit measures and the relative amount of work involved for in situ data collection (e.g., degree of physical difficulty involved in taking specific shoot measurements inside the canopy). We used predicted and observed DWS as data sets to which we fit linear models. Slope and intercepts of these models were tested to see if they were significantly different from the slope and the intercept of the 1:1 correspondence line (Dent and Blackie, 1979).

Leaflet angle and orientation are easily changed when a leaf is grabbed for measurement while still attached to the plant, thus changing W. Because LDW measurements on compound leaves of roses were subject to errors and were time consuming, only models using L were selected. Using this same practicality criterion, models for SDW and FDW determinations involved only one variable; developing fruit diameter was not used because measurement was difficult and time consuming.

\section{Results and Discussion}

Using the criteria delineated, we selected certain models (Table 1). LDW increased nonlinearly as the leaf lengthened (Fig. 1A). DW varied greatly, even for leaves of the same length; the variation became more pronounced for leaves $>170 \mathrm{~mm}$. These larger leaves represent an extreme not common on 'Cara Mia' flowering shoots. For 0-to 25-mm leaves, the model predicted (unrealistically) decreasing LDWs with increasing L. Such leaves are either bracts at the stem base or young unfolded leaves at the top of the flowering shoot. Because bracts and young leaves represent a small proportion of shoot LDW in a crop, it is a minor error in the predictions.

Although the model seems to be unbiased (Fig. 1B), the regression line of predicted vs. observed values has slope and intercept values significantly different $(P \leq 0.05)$ from those of the $1: 1$ correspondence line. Nevertheless, the acceptability of the model can be shown by comparing observed and predicted TLDWs of the 90 flowering shoots (Fig. 2). These TLDW values were calculated omitting leaves $<25$ $\mathrm{mm}$. The regression line of predicted vs. observed values is not significantly different from the 1:1 correspondence line. Differences between predicted and observed values may be due to the FDW variability previously mentioned. Possible causes of these differences could be natural plant variability, different amount and size of leaf thorns (found in the lower rachis), and differences in leaf thickness.

The $\mathrm{SDW}_{\mathrm{i}}$ increased nonlinearly with increasing $\mathrm{S}_{\mathrm{i}}$ (Fig. 3A). The variability in SDW of similar or equal diameter was high for all $\mathrm{S}_{\mathrm{i}} \mathrm{s}$. An error source could be that, for some shoots, the stem cross sections changed from circular to elliptical along the stem length. Depending on which diameter of the ellipse was used, estimated SDW varied considerably. Different number and size of thorns not only affected $\mathrm{SDW}_{\mathrm{i}}$ but also was an error

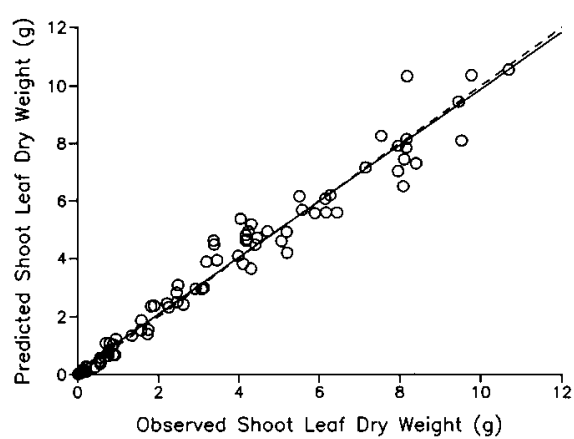

Fig. 2. Dry weight values of all leaves from a flowering shoot as a function of observed values. The dashed line represents the 1:1 correspondence and the solid line represents the linear regression of the predictions: $\mathrm{Red}=0.14+$ 0.97 Ohs.
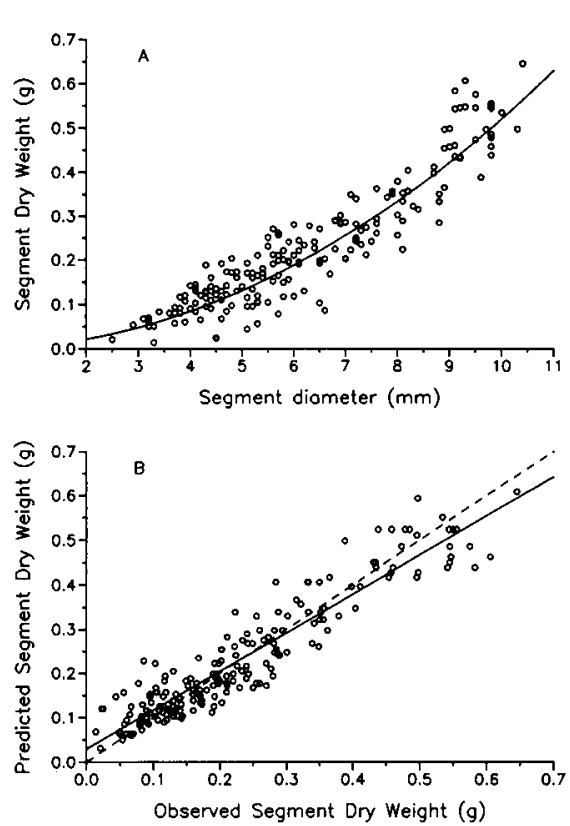

Fig. 3. (A) Observed (open circles) and predicted (solid line) stem segment dry weights $\left(\mathrm{SDW}_{\mathrm{i}}\right.$ ) as a function of stem segment diameter and (Et) predicted vs. observed stem segment dry weights. The dashed line represents the 1:1 correspondence and the solid line represents the linear regression of the predictions: Pred $=0.029+$ 0.887 Obs. source in diameter determination. The signification level that can affect the $\mathrm{SDW}_{\mathrm{i}}$ of equal diameter is not taken into account by this model and could represent a source of predictive error.

The regression line of the predicted vs. observed $\mathrm{SDW}_{\mathrm{i}}$ (Fig. 3B) is significantly different from the 1:1 correspondence due to overprediction of light segments and underprediction of heavier segments. However, the regression line of the predicted vs. observed SDW for the eight shoots used in data collection (Fig. 4) was not significantly different from the 1:1 correspondence line. The model's predictive capability seems to be good, although data from more shoots are needed to increase the degree of confidence in the model.

FDW increased nonlinearly with F (Fig. $5 \mathrm{~A})$. Variability in FDW increased with bud

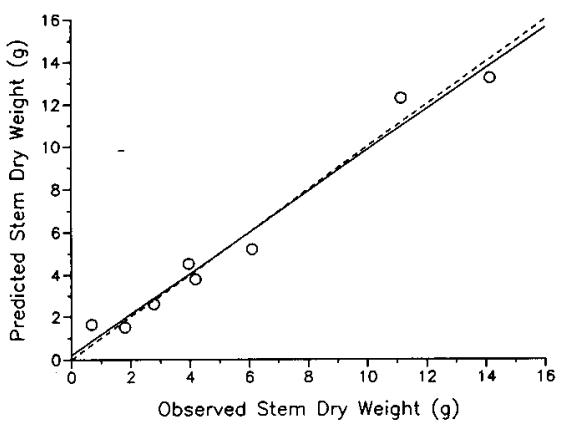

Fig. 4. Stem dry weight predicted as a function of observed values. The dashed line represents the 1:1 correspondence and the solid line represents the linear regression of the predictions: Pred $=$ 0. $194+0.97$ Obs
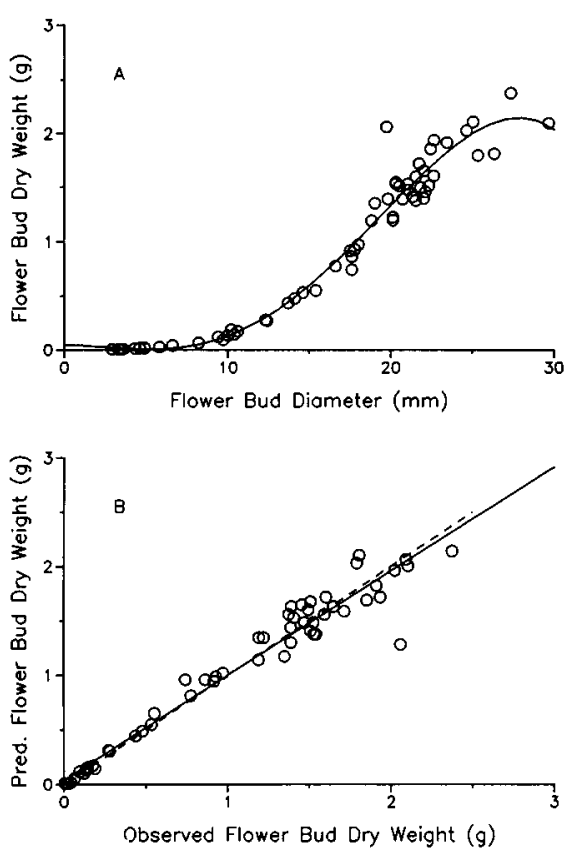

Fig. 5. (A) Observed (open circles) and predicted (solid line) flower bud dry weight as a function of flower bud diameter and (B) predicted vs. observed flower bud dry weights. The dashed line represents the 1:1 correspondence and the solid line represents the linear regression of the predictions: Pred $=0.041+0.958$ Ohs. 
diameter. However, variability for FDW was smaller than for LDW or $\mathrm{SDW}_{\mathrm{i}}$ (compare Figs. 1A, 3A, 5A). Some flower buds with 20- to 25 $\mathrm{mm}$ diameters and all those with diameters $>25 \mathrm{~mm}$ had horizontally positioned sepals (buds ready to harvest) that increased the diameter measurement error. Another error source was the presence of larger than normal leaf-like sepals in some buds.

The FDW model predicts decreasing values as $\mathrm{F}$ increases beyond $25 \mathrm{~mm}$. This is because of the empirical nature of the fourthpower polynomial. However, flowers with $\geq 25$ $\mathrm{mm}$ diameter are at or beyond the harvest point, with sepals detached from the unfolding petals. At this developmental stage, FDW predictions require a different model based on a different set of flower dimensions. Because our objective was to predict FDW up to harvest, the fourth-power polynomial was selected; it was superior to the quadratic and cubic powers for flowers with $<25$-mm diameters. The predicted values are close to the observed values, and the regression line of the predicted vs. observed values was not significantly different from the 1:1 correspondence line (Fig. 5B).

Although these methods have limitations, the models satisfy the subjective and difficultto-quantify practical measurement criteria and should be useful for crop ecologists and crop modelers interested in estimating greenhouse rose DW distribution over time. This is the only method feasible in commercial settings where destructive sampling would not be tolerated. Some caution should be taken before using this method because our predicted vs. observed plots show large errors in some cases. How much error can be tolerated and the availability and affordability of alternative methods should be considered before using this technique.
As with all regression models, dry-matter prediction for plant material beyond the sizes used for model development should be avoided.

\section{Literature Cited}

Dent, J.B. and M.J. Blackie. 1979. Systems simulation in agriculture. Applied Sci. Publ., London. Hoagland, D.R. and D.I. Arnon. 1950. The water culture method for growing plants without soil. California Agr. Expt. Sta. Circ. 347.

Ondark, J.P. 1971. Indirect estimation of primary values used in growth analysis, p. 392-411. In: Z. Sesták, J. Catsky, and P.G. Jarvis (eds.). Plant photosynthetic production: Manual of methods. Dr. Junk Publ., The Hague, The Netherlands.

Peace, W.J.H. and P.J. Grubb. 1982. Interaction of light and mineral nutrient supply in the growth of Impatiens parviflora. New Phytol. 90: 127150.

Statistical Analysis System. 1.987. SAS/STAT: Guide for personal computers, version 6 edition. SAS Institute, Inc., Cary, N.C. 